\title{
Quality care outcomes following transitional care interventions for older people from hospital to home: a systematic review
}

\author{
Jacqueline Allen ${ }^{1 *}$, Alison M Hutchinson ${ }^{2}$, Rhonda Brown $^{1}$ and Patricia M Livingston ${ }^{3}$
}

\begin{abstract}
Background: Provision of high quality transitional care is a challenge for health care providers in many western countries. This systematic review was conducted to (1) identify and synthesise research, using randomised control trial designs, on the quality of transitional care interventions compared with standard hospital discharge for older people with chronic illnesses, and (2) make recommendations for research and practice.

Methods: Eight databases were searched; CINAHL, Psychinfo, Medline, Proquest, Academic Search Complete, Masterfile Premier, Soclndex, Humanities and Social Sciences Collection, in addition to the Cochrane Collaboration, Joanna Briggs Institute and Google Scholar. Results were screened to identify peer reviewed journal articles reporting analysis of quality indicator outcomes in relation to a transitional care intervention involving discharge care in hospital and follow-up support in the home. Studies were limited to those published between January 1990 and May 2013. Study participants included people 60 years of age or older living in their own homes who were undergoing care transitions from hospital to home. Data relating to study characteristics and research findings were extracted from the included articles. Two reviewers independently assessed studies for risk of bias.

Results: Twelve articles met the inclusion criteria. Transitional care interventions reported in most studies reduced re-hospitalizations, with the exception of general practitioner and primary care nurse models. All 12 studies included outcome measures of re-hospitalization and length of stay indicating a quality focus on effectiveness, efficiency, and safety/risk. Patient satisfaction was assessed in six of the 12 studies and was mostly found to be high. Other outcomes reflecting person and family centred care were limited including those pertaining to the patient and carer experience, carer burden and support, and emotional support for older people and their carers. Limited outcome measures were reported reflecting timeliness, equity, efficiencies for community providers, and symptom management.
\end{abstract}

Conclusions: Gaps in the evidence base were apparent in the quality domains of timeliness, equity, efficiencies for community providers, effectiveness/symptom management, and domains of person and family centred care. Further research that involves the person and their family/caregiver in transitional care interventions is needed.

Keywords: Transitional care, Discharge care, Discharge planning, Older person care, Aging, Systematic review

\footnotetext{
* Correspondence: jacqui.allen@deakin.edu.au

'Deakin University, School of Nursing and Midwifery, 221 Burwood Hwy,

Burwood 3125, Vic, Australia

Full list of author information is available at the end of the article
}

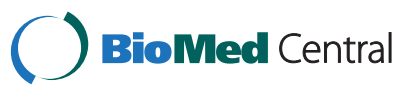

(c) 2014 Allen et al.; licensee BioMed Central Ltd. This is an Open Access article distributed under the terms of the Creative Commons Attribution License (http://creativecommons.org/licenses/by/4.0), which permits unrestricted use, distribution, and reproduction in any medium, provided the original work is properly credited. The Creative Commons Public Domain Dedication waiver (http://creativecommons.org/publicdomain/zero/1.0/) applies to the data made available in this article, unless otherwise stated. 


\section{Background}

Older people with complex comorbid health problems are frequently required to transition between hospital and home during an episode of acute illness. Estimates from the United States suggest that at least $20 \%$ of older Medicare recipients with five or more chronic conditions require frequent inpatient and emergency care from hospitals $[1,2]$. The provision of high quality transitional care continues to be a challenge in many western countries because of a continued focus on acute, episodic care [3-7]. In Western countries, health care quality standards and expectations emphasise effective, efficient, safe, timely and equitable care in addition to person and family centred care [4,6-8]. However, previous researchers have found that studies investigating transitional care interventions have focussed on re-hospitalization rates and cost containment for inpatient providers [9-12]. This suggests that other indicators of quality of care have received less attention. These indicators include other domains of care effectiveness, efficiency and safety: for example; symptom management, self-management and efficiencies for community providers. Additionally, quality indicators of timeliness, equity and person and family centred care have received limited focus in research outcomes. This review was conducted to synthesise the evidence in relation to quality outcomes following transitional care for older people and their caregivers transferring from hospital to home in order to make recommendations for research and practice.

\section{Demand on health services}

The frequency and complexity of care transitions for older people is expected to increase considerably along with the predicted increases in demand on health and aged care services [13]. Globally, populations are aging due to declines in fertility and increases in life expectancy [14-16]. One effect of the aging population is the growing numbers of older people living with chronic illness who are expected to require extensive health and aged care from multiple providers and across multiple care settings $[13,17,18]$. Some policy makers and health planners in Australia have predicted that the numbers of people in the future workforce will be unable to sustain the resources required to support older people $[5,13]$. There is a risk, however, that these views may be used to justify suboptimal health and aged care for older people [19]. Importantly, societies can adapt to changes in population aging and adopt age inclusive policies and practices $[16,19]$.

Quality in health care standards and indicators recommended in the United States of America [4], United Kingdom [8] and Australia [6] (see Table 1) include: effectiveness, efficiency, safety and risk, timeliness, equity and person and family centred care, offer opportunities and guidance for optimal health and aged care for older
Table 1 Definition of quality indicators

\begin{tabular}{ll}
\hline Quality indicator & Definition of indicator \\
\hline Effectiveness & $\begin{array}{l}\text { Effective care is based in evidence and } \\
\text { is provided to the people most likely } \\
\text { to benefit }[4,6,8] .\end{array}$ \\
Efficient care is care without waste, \\
including wasted resources [4]. \\
Timely care is care that is provided in \\
Timeliness & $\begin{array}{l}\text { a timely manner without lengthy } \\
\text { their family [4]. }\end{array}$ \\
Safety and risk & Care is low risk and safe when it causes \\
& no harm to patients, families or health \\
care staff $[4,6,8]$. \\
Care that is fair to everyone. No group \\
of people receive inferior care based in \\
differences in gender, culture, ethnicity, \\
age, sexuality, geographic location or \\
socioeconomic status [4]. \\
Care that is respectful of patients and \\
families preferences, values and goals. \\
Care decisions involve patients and \\
families [4,6,8].
\end{tabular}

people including optimal transitional care from hospital to home. Research in the field of transitional care that is focussed on quality indicators is vital in guiding age centred policies and practices.

\section{Transitional care}

Transitional care is a broad term for care interventions that promote safe and timely transfer of patients between levels of care and across care settings [20-23]. Transitional care is not strictly defined by beginning and end points; it includes pre hospital discharge activities and immediate post hospital discharge follow-up at the next location of care $[21,24]$. Transitional care can be considered a part of integrated care, which occurs over longer duration of care episodes [25] and it can be considered as a part of prevention of re-hospitalization programs within longer-term chronic disease management initiatives [26]. Although transitional care is related to integrated care and prevention of re-hospitalization programs, it is considered a conceptually distinct category of care interventions [24]. According to Coleman and Boult [20], there are a number of essential elements in quality transitional care: communication between providers about the discharge assessment and plan of care, preparation of the patient and carer for the care transition, reconciliation of medications at transition, a plan for follow-up, and patient education about self-management.

Preventable adverse events, including medication errors, falls, errors in diagnosis, post-operative infections and confused states, are risks for older people during care transitions, particularly those with functional difficulty and chronic illness [11,27]. Consequently, discharge planning 
that occurs solely within the acute inpatient setting without in-home follow-up support is not sufficient in the case of many older people with chronic illnesses and functional difficulty $[22,25,28]$. The success of care transitions for many older people depends on holistic transitional care interventions involving both hospital discharge planning and in-home follow-up and support [13,29].

\section{What is currently known about quality transitions?}

Reviews of the literature in transitional care interventions have focussed on assessing outcomes of re-hospitalization and length of stay with mixed findings [24,27,30-33]. Although in a recent Cochrane systematic review of transitional care nested within disease focussed models, Shepperd and colleagues [12] concluded that transitional care was "probably" effective in reducing rates of rehospitalization in the immediate post discharge period and reducing length of stay. Shepperd and associates [12] further concluded that cost was most likely shifted from the inpatient to the community sector.

Risk has also been a focus in the literature. Mansah and colleagues [27] found that pharmacist led interventions in medication reconciliation reduced adverse events associated with non-adherence with medications in the home. Laugaland and colleagues [11] further identified transitional care interventions that reduced adverse events post discharge as those that commenced early in hospitalisation, involved key workers/discharge coordinators, included patients and family carers, involved a multidisciplinary and multicomponent approach, and reconciled medications. Some reviewers nominated re-hospitalization rates as an outcome capturing risk and safety following transitional care $[27,30,34]$. Effective communication between health providers during care transitions of older people has also been identified as important in reducing risks and adverse outcomes [11,27,34,35].

Numerous reviewers have identified limited research and mixed findings about person and family centred experiences during care transitions and outcomes following transitional care interventions [10,12,24,31,32,35-37]. These findings indicate that the older person's experience and the experiences of their family/carer have not received sufficient attention in the transitional care intervention research to date.

Transitional care for older people has been evaluated largely in terms of re-hospitalization rates, thereby capturing specific dimensions of quality such as effectiveness, efficiency for inpatient providers, and risk and safety [27,30]. Other important dimensions of quality in health care; person and family centred care, symptom management, efficiencies for community providers, timeliness and equity, have not received the same focus. Additionally, a research emphasis on reducing rates of re-hospitalization may unintentionally and subtly contribute to the exclusion of older people from health care $[5,13,16,19]$. A holistic understanding of quality of care transitions is therefore required if transitional care providers and researchers are to assist societies and health care systems to adapt positively to changes associated with population aging.

\section{Objectives}

This systematic review was conducted to:

(1) Locate and synthesise research using randomised control trial designs on quality of outcomes following transitional care interventions compared with standard hospital discharge for older people with chronic illnesses.

(2) Make recommendations for research and practice.

\section{Methods}

This systematic review synthesised published studies, using randomised controlled trial designs, to investigate the effects of transitional care interventions for people aged 60 years and older on health care outcomes. Cochrane Collaboration guidelines [38] were used to direct the review.

\section{Search strategy}

A search for peer-reviewed journal articles was conducted using the search terms: 'discharge planning', ' hospital discharge', 'discharge care pathways', 'discharge care protocols', 'transitional care', 'transitional care pathways', 'transitional care protocols'. These terms were added to the phrase 'from the inpatient setting to the home' to form concept groups. These concept groups were further combined with 'aged care' and similar terms (aging, geriatrics, gerontology and older person care), and 'community' and similar terms (home care, primary care, domiciliary care). Eight databases were searched: CINAHL, Psychinfo, Medline, Proquest, Academic Search Complete, Masterfile Premier, SocIndex, Humanities and Social Sciences Collection, in addition to the Cochrane Collaboration, Joanna Briggs Institute and Google Scholar.

Studies were limited to those published between January 1990 and May 2013. This timeframe was chosen due to the development since the 1990s in many western countries of community-based care programs and the evolution of more formally structured transitional care interventions inclusive of discharge processes and in-home follow-up $[21,39,40]$.

\section{Inclusion criteria}

To be included, an article was required to (1) be published in a peer reviewed journal, (2) report on a transitional care intervention compared with standard hospital discharge, (3) use a randomized control trial design, (4) be published in English, and (5) provide an analysis of outcomes that evaluated quality indicators related to older people. 
Transitional care included any intervention applied in the inpatient setting, inclusive of follow-up in the community. All studies included people 60 years of age or older. Sixty years of age was selected because it was the definition of 'older adult' used by the World Health Organization $[13,41]$.

\section{Screening procedure}

Articles were entered into an Endnote version 16 database [42] for screening and duplicates were removed. Two reviewers independently screened the title and abstract of each study to identify articles meeting the inclusion criteria. Records for which relevance could not be determined based on title and abstract alone were screened from the full text journal article. Discrepancies in reviewers' decisions regarding relevance for inclusion were resolved by consensus.

\section{Data extraction}

Additionally, a data extraction tool was devised, based on an earlier literature review [9], to capture the main features of studies meeting the inclusion criteria (see Additional file 1). A single reviewer extracted the data for all included studies. Due to the heterogeneity in the transitional care interventions and outcomes, data were presented in tables and were not pooled.

\section{Assessment for risk of bias}

The Cochrane Collaboration's tool for the assessment of bias in randomized controlled trials was used to assess for bias in all included studies [38]. This tool appraises numerous areas of potential bias; selection bias, performance bias, detection bias, attrition bias, reporting bias and 'other' sources of bias. The potential for selection bias is assessed in terms of the adequacy of randomisation processes (random sequence generation) and the adequacy of the concealment of allocation to intervention group (allocation concealment) [38]. Performance bias is the study bias that may result from the knowledge of research participants and research staff of the interventions that participants were allocated to. Detection bias is possible when outcome assessors know which interventions participants were allocated to [38]. Attrition bias is the potential for biased conclusions resulting from incomplete outcome data. Reporting bias may result from the selection of particular outcomes for reporting [38]. The potential for other sources of bias (other bias) was also appraised. Two reviewers independently assessed included studies for study bias in accordance with the guidelines for the bias assessment tool [38]. Both reviewers then met to compare their findings. Consistency rates between reviewers were high ( $>80 \%$ ) with minor discrepancies resolved through discussion with a third reviewer.

\section{Results}

The search identified 405 records. Of these, 12 published journal articles met the inclusion criteria. Outcomes from the search and screening results are presented in Figure 1.

Title and abstract screening resulted in the exclusion of 368 articles. Full texts for 37 articles were retrieved and a further 25 articles were excluded. Reasons for the exclusion of these articles are presented in Table 2.

In total 5,269 older people were included across 12 randomised controlled trial studies conducted in four western countries: USA (7 studies), Australia (3 studies), Denmark (1 study), and France (1 study).

\section{Transitional care interventions}

Each of the transitional care interventions tested in the 12 studies [66-77] contained elements considered essential to high quality transitional care: discharge assessment and care planning, communication between providers, preparation of the person and carer for the care transition, reconciliation of medications at transition, community-based follow-up, and patient education about self-management $[20,24]$. The main practitioner/s responsible for implementing the transitional care intervention varied across the 12 studies. Advanced practice nurses (nurses educationally prepared at Masters degree level) implemented the transitional care in five studies $[68,72,74,75,77]$. General practitioners (physicians in primary care) and primary care nurses (nurses educationally prepared at either Bachelor degree level or diploma level in primary care, also referred to as practice nurses) implemented transitional care in three studies $[66,67,73]$. The older person and their carer implemented their own transitional care with the support of a transition coach in the study by Coleman et al. [69]. Case managers were responsible for care transitions in the study by Lim et al. [76] and geriatricians were responsible for transitional care in the studies by Hansen et al. [70] and Legrain et al. [71].

With the exception of the self-management and transition coaching intervention described by Coleman et al. [69] there was limited reporting on the involvement of older people and their carers/family in the development of the transitional care intervention. Coleman et al. [69] reported that their intervention was informed by focus groups with older people and their families/carers who articulated what was important to them in quality care transitions and what sort of assistance they wanted in these care episodes [64].

The main limitations identified across the 12 studies were in relation to the generalizability of findings. Findings would only be generalizable to those people with similar characteristics to those included in the sample and to the practitioners implementing the intervention.

Characteristics of the included studies are presented in Table 3. 


\section{Outcomes}

Table 4 presents a summary of the outcome findings of the randomised controlled trial studies. Table 5 presents a summary of quality indicators measured in randomised controlled trial study outcomes.

\section{Efficiency, effectiveness and safety}

Re-hospitalization rates, length of stay, and costs are considered important indicators of efficiency, effectiveness and patient safety $[4,6]$. Eleven of the twelve studies measured re-hospitalization rates following the transitional care intervention [67-77], and three studies measured length of stay [66,73], [86]. In six studies, significant reductions in re-hospitalization rates were found for people in the intervention groups at up to six months following hospital discharge [68-70,72,75,77] and at up to three months following discharge in the study by Legrain et al. [71].
Three studies did not find any difference in rehospitalization rates between treatment and control groups at up to six month follow up $[73,74,76]$. One study by Weinberger et al. [67] found the veterans in the intervention group had significantly higher rates of re-hospitalization than veterans in the control group. Weinberger et al. [67] speculated that the veterans in their study were experiencing very poor health and that the transitional care intervention assisted in early identification of health difficulties requiring re-hospitalisation.

Lim et al. [76] found reduced length of stay when older people were re-admitted following the intervention. Two studies [66,73] found no significant differences in length of stay between intervention and control groups. One study by Weinberger et al. [67] found the veterans in the intervention group who were re-admitted had a longer stay in hospital than veterans in the control group. 
Table 2 Reason for exclusion for studies retrieved in full text $(n=25)$

\begin{tabular}{|c|c|}
\hline First author (year) & Reason for exclusion \\
\hline Arbaje (2010) [43] & Quasi-experimental design \\
\hline Bull (2000) [44] & $\begin{array}{l}\text { Not a full transitional care intervention, } \\
\text { discharge planning }\end{array}$ \\
\hline Balaban (2008) [45] & $\begin{array}{l}\text { Adult sample, not focussed on older } \\
\text { people with functional difficulty }\end{array}$ \\
\hline Bonnet-Zamponi (2013) [46] & $\begin{array}{l}\text { Secondary data analysis, drug related } \\
\text { problems }\end{array}$ \\
\hline Brand (2004) [47] & Quasi-experimental design \\
\hline Coleman (2004) [48] & Quasi-experimental design \\
\hline Dedhia (2009) [49] & Quasi-experimental design \\
\hline Einstadter (1996) [50] & $\begin{array}{l}\text { Not a full transitional care intervention, } \\
\text { discharge planning }\end{array}$ \\
\hline Golden (2010) [51] & $\begin{array}{l}\text { Not a full transitional care intervention, } \\
\text { discussion paper }\end{array}$ \\
\hline Haggmark (1997) [52] & $\begin{array}{l}\text { Not a full transitional care intervention, } \\
\text { interprofessional care }\end{array}$ \\
\hline $\operatorname{Ham}(2011)[3]$ & $\begin{array}{l}\text { Not a full transitional care intervention, } \\
\text { integrated care }\end{array}$ \\
\hline Hansen (1992) [53] & Not a full transitional care intervention \\
\hline Hebert (2008a) [54] & $\begin{array}{l}\text { Not a full transitional care intervention, } \\
\text { integrated care }\end{array}$ \\
\hline Hebert (2008b) [55] & $\begin{array}{l}\text { Not a full transitional care intervention, } \\
\text { integrated care }\end{array}$ \\
\hline Hegney (2002) [56] & $\begin{array}{l}\text { Not a full transitional care intervention, } \\
\text { discharge planning }\end{array}$ \\
\hline Jack (2009) [57] & $\begin{array}{l}\text { Adult sample, not focussed on older } \\
\text { people with functional difficulty }\end{array}$ \\
\hline Jeangsawang (2012) [58] & $\begin{array}{l}\text { Not randomised controlled trial or } \\
\text { quasi-experimental design }\end{array}$ \\
\hline Lattimer (2012) [59] & Discussion paper \\
\hline Melton (2012) [60] & $\begin{array}{l}\text { Adult sample, not focussed on older } \\
\text { people with functional difficulty }\end{array}$ \\
\hline O'Reilly (2008) [61] & Rehabilitation, not transitional care \\
\hline Ornstein (2010) [62] & $\begin{array}{l}\text { Mixed methods, not randomised } \\
\text { controlled trial, not quasi-experimental } \\
\text { design }\end{array}$ \\
\hline Parker (2004) [30] & Systematic review \\
\hline Parker (2009) [63] & Rehabilitation, not transitional care \\
\hline Parry (2008) [64] & $\begin{array}{l}\text { Psychometric study, cross sectional } \\
\text { design }\end{array}$ \\
\hline Steeman (2006) [65] & Quasi-experimental design \\
\hline
\end{tabular}

Two studies assessing the effectiveness of general practitioner and practice nurse interventions on rehospitalization rates $[67,73]$ or on length of stay $[66,73]$ did not find significant improvements in these outcomes.

Costs were assessed in three studies $[68,75,77]$. In each of these studies [68,75,77], costs were reduced for those people who received the intervention. Efficiencies for community providers were assessed in only one study.
Enguidanos et al. [74] found fewer visits to general practitioners were required for those people who received the intervention.

Other quality indicators were assessed to determine the effectiveness of transitional care. Of the 12 included studies, two studies addressed functional status (Naylor et al. [77] and Naylor et al. [75]). Neither study found statistically significant differences on these measures for people who received the intervention. The study by Naylor et al. [77] assessed depressive symptoms following the intervention and found no statistically significant differences between intervention and control groups. Although assessment of re-hospitalization rates is inclusive of symptom control, few studies specifically measured symptom management following discharge or transitional care.

Quality of life was assessed in four studies $[66,67,75,76]$. Naylor et al. [75] found an improvement for people who had participated in the intervention in physical quality of life. Preen et al. [66] found a significant improvement in mental quality of life for people who received the intervention one week following discharge. Lim et al. [76] found quality of life was better in people who had participated in the intervention at one-month follow-up. Weinberger et al. [67] found no differences in quality of life scores between veterans in their intervention and control groups.

\section{Person and family centred care}

Person and family centred care is considered essential to the quality of health care provision [4]. Patient satisfaction was measured in six of the 12 identified studies [66,67,73-75,77]. Naylor et al. [75], Weinberger et al. [67], McInnes et al. [73], and Preen et al. [66] found that patient satisfaction scores for older people in the intervention groups were significantly improved compared with standard hospital discharge. Naylor et al. [77] and Enguidanos et al. [74] found no improvements in patient satisfaction following implementation of the transitional care intervention. Caregiver burden was measured in two studies [68,76]. Naylor et al. [68] and Lim et al. [76] found no change in caregiver burden at one-month follow-up.

\section{Timeliness and equity}

Timeliness and equity are the two remaining quality indicators recommended by the Institute of Medicine [4]. Of the 12 studies, one study assessed timeliness. Preen et al. [66] found general practitioners reported satisfaction with the timely communication resulting from the intervention. Three studies [66,70,73] assessed equity and access to services and found that people in their intervention groups were more likely to be referred to community-based services. 
Table 3 Study characteristics - included studies $(n=12)$

\section{First author (year) Setting, sample \& study design}

Discharge protocol \& advanced practice nurse

\section{Naylor (1990) [72] US, acute inpatient (medical, surgical)}

to home

$N=40$, average age 78.8 years

Chronic illness

RCT

Naylor (1994) [68]

US, acute inpatient (medical, surgical) to home

$N=276$, average age 76 years

Chronic illness

$\mathrm{RCT}$

Naylor (1999) [77]

US, acute inpatient (medical, surgical) to home

$N=363$, average age 75.4 years

Chronic illnesses

$\mathrm{RCT}$

Naylor (2004) [75]
To assess an APN implemented discharge planning protocol compared with standard hospital discharge

protocol of discharge planning compared with standard hospital discharge

To assess an APN implemented compared with standard hospital of re-hospitalization

To assess the effects of an advanced practice nurse delivered transitional care intervention on older people with heart failure and comople with heart falu conditions
Intervention

Limitation

Protocol implemented by advanced practice nurses (APN):

Assessment and discharge planning within 24 hours of admission

Discharge plan included health teaching to be conducted in primary care

APN telephone follow up for 2 weeks post discharge

Discharge planning protocol implemented by APNs:

Discharge assessment 24-48 hours after hospital admission

Discharge plan developed collaboratively with client, carer, multidisciplinary team

Communication and coordination maintained by APN throughout this process with multidisciplinary team including primary care providers

Post discharge APN phone availability APN protocol discharge planning and home support follow up:

APN care continuity

APN conducted hospital discharge planning care and in home support (substituted for the visiting nurse) for the first 4 weeks post discharge

APN individualised patient care in collaboration with the person's physician

Advanced practice nurse conducted a transitional care intervention emphasising

Discharge assessment

Discharge plan

Discharge coordination with

multidisciplinary team

APN care continuity

\section{Education}

Symptom management and self-management
Small sample size

Costs of nursing intervention was incomplete due to missing data

Generalizability of findings is limited to older people with cardiovascular diagnoses, oriented and alert at admission, well educated, with good support systems and few functional deficits
Generalizability of findings is limited to older people oriented and alert at admission

Intervention may be limited to deployment by advanced practice nurses in primary care

Generalizability of findings is limited to older people with exacerbation of cardiac failure and co morbid conditions

Intervention may be limited to deployment by advanced practice nurses 
Table 3 Study characteristics - included studies $(n=12)$ (Continued)

\section{$\mathrm{RC}$}

Enguidanos (2012) [74] US, acute care to home

$\mathrm{N}=199$, average age 73.58 years

Chronic illnesses

$\mathrm{RCT}$

General practitioner and primary care nurse models

Weinberger (1996) [67] US, acute care to home

$N=1396$, average age 63 years, veteran sample, mostly male (98.5\%)

Chronic illnesses

$\mathrm{RCT}$

McInnes (1999) [73] Australia, acute hospital-geriatric care unit (patients admitted under care of geriatrician) to home

$N=364$, average age 81 years
To assess impact of brief nurse practitioner (NP) intervention for older people discharged from hospital to home compared with standard hospital discharge

To test an primary care intervention on rates of re-hospitalization, length of stay, quality of life and veteran satisfaction compared with standard discharge care

To test if GP involvement in discharge planning patient outcomes when compared with standard hospital discharge
Goal setting

Medication management

Home visits/home nursing up to

3 months following discharge

NP in primary care conducted:

Education about discharge instructions

to older person

Medication reconciliation

Sample size insufficiently powered to detect an effect of the intervention

Intervention may be limited to deployment by nurse practitioners

Home care needs assessment and referral to resources

scheduling follow-up medica appointments

Before discharge:

The primary care nurse conducted the discharge assessment, provided educatio and the contact telephone numbers of the primary care nurse and general practitioner (GP), and scheduled an appointment within 2 days of discharge to attend the primary care clinic

The GP visited the veteran in hospita within 2 days prior to discharge and reviewed the discharge plan, medication, and medical problems with the hospital physicians

After discharge:

The primary care nurse telephoned the patient (within 2 working days of discharge) at home to assess any difficulties with medications/medical treatments, health problems, remind of follow-up appointment

Patients were followed-up in clinic

The primary care nurse and GP reviewed treatment plan at first appointment.

Standard hospital discharge practice with the addition of GP visit pre discharge:

GPs invited to undertake pre discharge visit:

Of those randomized to the intervention group only $52 \%$ of patients were actually visited by their GP in hospital

Substantial primary care resources were required of study limited to older US male veterans

Substantial primary care resources were required to implement the interventio 
GP able to discuss care/treatment with

hospital based medical and allied

health staff

GP had access to the patient's hospital care record during the visit

Preen (2005) [66]_Australia, acute hospital to home

$\mathrm{N}=189$, average age 75 years

Chronic illnesses

$\mathrm{RCT}$

Self-management and transition coaching

Coleman (2006) [69] US, acute hospital to home

$N=747$, average age 76 years

Chronic illnesses

$\mathrm{RCT}$

\section{Discharge case management}

Lim (2003) [76]

Australia, acute hospital to home

$N=598$, average age 76 years

Chronic illnesses
To test a hospital coordinated discharge plan that involved the GP when compared with standard hospital discharge

To assess the effects of a care transitions intervention in

comparison with standard hospita discharge care, using RCT design, on rehospitalisation rates for older people

Research nurse based in the hospital:

Developed discharge plan (determined

client discharge problems, goals and

community service provider involvement)

Faxed the discharge plan to the GP 24-48

hours prior to discharge

The GP

Reviewed the discharge plan, modified it and returned it to the hospital by fax

Research nurse based in the hospita:

Explained the discharge plan to the client

Provided copies of the discharge plan to

the client, and all service providers

identified on the care plan.

Scheduled an appointment with the GP

Care Transitions Intervention (as per Coleman et al. 2004 above) Intervention developed from qualitative research with older people and their care givers about what would be most valuable to them

during care transitions:

Medication assistance and self-management

Patient centred and owned record

Timely follow-up from primary care providers

List of problem triggers indicating deterioration in their particular chronic illness and what to

do about these

To test the effects of case

management and post acute care services on organisation and patien

Post Acute Care program:

Short term case management and provision of post-acute care services (in home) nursing allied health, community supports
Intervention was not fully implemented as only $42 \%$ of GPs returned the discharge plan to the hospital prior to discharge

Sample size may have been insufficiently powered to detect an effect of the intervention

Intervention may be limited to deployment by advanced practice nurses in the role of transition coach

Costs were averages of community services and daily hospital bed utilisation rates, actual costs for each individual were not captured 
Table 3 Study characteristics - included studies $(n=12)$ (Continued)

\begin{tabular}{|c|c|c|c|c|}
\hline & $\mathrm{RCT}$ & \multicolumn{2}{|l|}{$\begin{array}{l}\text { caregiver outcomes in comparison } \\
\text { with standard hospital discharge }\end{array}$} & \\
\hline \multicolumn{5}{|c|}{ Inpatient geriatric evaluation, co-management (with ward staff) and transitional care } \\
\hline \multirow[t]{4}{*}{ Hansen (1995) [70] } & $\begin{array}{l}\text { Denmark, subacute geriatric ward to } \\
\text { home }\end{array}$ & \multirow{4}{*}{$\begin{array}{l}\text { To compare the intervention with } \\
\text { standard hospital discharge on the } \\
\text { number of medical and social } \\
\text { problems after discharge, the } \\
\text { need for modification of the } \\
\text { discharge plan after discharge } \\
\text { and rates of re-hospitalization } \\
\text { to hospital }\end{array}$} & \multirow{2}{*}{$\begin{array}{l}\text { The Geriatric Evaluation and } \\
\text { Management team (geriatrician, nurse } \\
\text { and physical therapist) supported } \\
\text { inpatient discharge planning and } \\
\text { follow-up at home }\end{array}$} & $\begin{array}{l}\text { Generalizability of findings limited to older } \\
\text { people with low functioning }\end{array}$ \\
\hline & $\begin{array}{l}N=193 \text {, average age intervention } 78 \\
\text { to } 80 \text { years }\end{array}$ & & & $\begin{array}{l}\text { Intervention may be limited to deployment } \\
\text { by geriatricians }\end{array}$ \\
\hline & $\begin{array}{l}\text { Multiple chronic conditions and low } \\
\text { functional status }\end{array}$ & & \multirow{2}{*}{$\begin{array}{l}\text { Follow-up involved re-evaluation and } \\
\text { modification of the care plan, } \\
\text { communication with the primary } \\
\text { care team (GP, community nurse) } \\
\text { during home visits at } 1,3,8,16 \text { weeks } \\
\text { following discharge }\end{array}$} & \\
\hline & RCT & & & \\
\hline \multirow[t]{8}{*}{ Legrain (2011) [71] } & \multirow{5}{*}{$\begin{array}{l}\text { France, acute inpatient geriatric care } \\
\text { unit to varying locations: home, nursing } \\
\text { home, rehabilitation unit, acute care unit }\end{array}$} & \multirow{5}{*}{$\begin{array}{l}\text { To compare a comprehensive } \\
\text { discharge intervention with } \\
\text { standard hospital discharge on } \\
\text { emergency department visits and } \\
\text { re-hospitalisations }\end{array}$} & $\begin{array}{l}\text { Geriatrician delivered inpatient } \\
\text { intervention: }\end{array}$ & \multirow[t]{3}{*}{$\begin{array}{l}\text { Findings generalizable to functionally } \\
\text { dependent older people }\end{array}$} \\
\hline & & & Medication review & \\
\hline & & & $\begin{array}{l}\text { Education re self-management of } \\
\text { disease }\end{array}$ & \\
\hline & & & Communication principally with GP & \multirow{5}{*}{$\begin{array}{l}\text { Intervention may be limited to deployment } \\
\text { by geriatricians }\end{array}$} \\
\hline & & & Screening for main risks for frail elderly & \\
\hline & \multicolumn{2}{|l|}{$\mathrm{N}=665$, average age 86 years } & Depression & \\
\hline & \multicolumn{3}{|l|}{ Chronic illnesses } & \\
\hline & \multicolumn{3}{|l|}{$\mathrm{RCT}$} & \\
\hline
\end{tabular}


Table 4 Main findings - included studies $(n=12)$

\section{First author (year) \\ Discharge protocol \& advanced practice nurse}

Naylor (1990) [72]

Naylor (1994) [68]

Naylor (1999) [77]

Naylor (2004) [75]

Enguidanos (2012) [74]

General practitioner and primary care nurse models Weinberger (1996) [67]

Mclnnes (1999) [73]

Preen (2005) [66]

\section{Main findings}

Significant reduction in rates of re-hospitalization for intervention group over the 12 weeks post discharge

No difference in length of stay

No difference in posthospital infections

Intervention patients in the medical units at 6 week follow-up experienced:

Significant delay in re-hospitalization to hospital

Fewer total days of re-hospitalisation

Lower health care costs (inclusive of inpatient, clinic, home visits)

No change in functional status, mental status, self-esteem or affect

Intervention caregivers up to 12 weeks following discharge experienced:

No change in functional status, caregiving demands, family

functioning, affect

Intervention group at 24 week follow-up experienced fewer:

Re-hospitalizations

Hospital days per patient

Lower costs than control group

No statistically significant differences in functional status, depression or patient satisfaction between groups

The time to first admission was longer in intervention patients

At 52 weeks, intervention patients had fewer re-hospitalizations and lower total mean costs

There were short term improvements among intervention patients in quality of life (physical domain, up to 12 weeks post discharge) and satisfaction with discharge and transition care (up to 6 weeks post discharge)

No change in re-hospitalization rates at 6 months following enrolment in the study The intervention group experienced significantly fewer visits to GPS

There were no changes between intervention and control groups in self-efficacy or satisfaction with service

At 6 months following discharge:

Intervention group had significantly higher rates of re-hospitalization and if re-admitted longer in hospital stay than controls (discharge as usual).

Intervention group were significantly more satisfied with their care than controls

No differences in quality of life scores between groups

Quality of life scores were low in both groups

At 6, 12, 26 weeks following discharge:

No significant differences in length of stay, rates of re-hospitalization or time to first re-hospitalization

Intervention patients were significantly more likely to be

Referred to community services at discharge and report that hospital staff had discussed their discharge plan with them

Intervention patients reported increased satisfaction with discharge arrangements and preparation

There were no differences in length of stay between groups

One week following discharge:

GPs in the intervention group were more satisfied with the documentation 


\begin{tabular}{|c|c|}
\hline & $\begin{array}{l}\text { Discharge communication to GPs in the intervention was significantly faster than for } \\
\text { GPs in the control group }\end{array}$ \\
\hline & $\begin{array}{l}\text { Patients in the intervention group reported improved satisfaction with discharge } \\
\text { planning, access to health services, confidence with discharge, and mental quality } \\
\text { of life }\end{array}$ \\
\hline Self-management anc & \\
\hline Coleman (2006) [69] & $\begin{array}{l}\text { Intervention group had significantly lower re-hospitalization rates than the control } \\
\text { group at 30, } 90 \text { and } 180 \text { days post discharge }\end{array}$ \\
\hline & $\begin{array}{l}\text { Intervention group had significantly lower hospital costs than the control group at } \\
30,90 \text { and } 180 \text { days post discharge }\end{array}$ \\
\hline Discharge case mana & \\
\hline $\operatorname{Lim}(2003)$ [76] & $\begin{array}{l}\text { Over } 6 \text { month follow-up period there were no differences in rates of unplanned } \\
\text { re-hospitalizations }\end{array}$ \\
\hline & Intervention patients had significantly reduced length of stay (index hospitalisation) \\
\hline & Costs (hospital utilisation) lower in intervention patients over 6 \\
\hline & months following discharge \\
\hline & No differences in costs (utilisation of community services) \\
\hline & between groups \\
\hline & $\begin{array}{l}\text { Significantly improved self-reported quality of life in intervention patients at one } \\
\text { month follow-up }\end{array}$ \\
\hline & No difference in caregiver burden at 1 month follow-up \\
\hline Inpatient geriatric ev & nd transitional care \\
\hline Hansen (1995) [70] & At 6 months following discharge: \\
\hline & $\begin{array}{l}\text { People in the intervention group were significantly less likely to be re-admitted to } \\
\text { hospital than those in the control group }\end{array}$ \\
\hline & There were no differences in rates of admission to nursing homes or mortality rates \\
\hline & $\begin{array}{l}\text { Significant increase in new and unforseen problems identified following discharge } \\
\text { in people receiving the intervention. }\end{array}$ \\
\hline & Intervention participants were significantly more likely to be allocated home help. \\
\hline Legrain (2011) [71] & $\begin{array}{l}\text { Older people in the intervention group were significantly less likely to attend the } \\
\text { emergency department or be re-admitted at } 3 \text { months following discharge }\end{array}$ \\
\hline & $\begin{array}{l}\text { There were no differences between groups in ED attendances or re-hospitalizations } \\
\text { at } 6 \text { months following discharge }\end{array}$ \\
\hline
\end{tabular}

\section{Bias assessment}

The potential for selection bias was assessed in terms of the adequacy of random sequence generation and allocation concealment [38]. Random sequence generation was identified as adequate with low risk of selection bias in nine of the 12 studies with three studies providing insufficient information about how the random sequence generation was conducted [66-68]. Allocation concealment was assessed as adequate with low risk of selection bias in eight of the 12 studies with four studies providing inadequate information about how this was undertaken $[66,68,69,70]$. In the Cochrane tool, performance bias is the potential bias resulting from knowledge of research participants and research staff of the interventions that participants were allocated to [38]. No studies were identified as low risk in regard to performance bias.
Detection bias, according to the Cochrane bias assessment tool, is the potential for bias resulting from outcome assessors' knowledge of the interventions that participants were allocated to [38]. Of the 12 studies, five provided insufficient information to assess the risk of detection bias as these studies did not report if the outcome data collectors were blinded to participant group [66,68,70-72]. The study by McInnes [73] was assessed to have low risk of detection bias for service utilisation outcomes but did not report how this risk was managed in relation to questionnaire data.

The risk of attrition bias, the potential for biased conclusions resulting from incomplete outcome data [38], was unclear across most of the included studies with exception to Coleman [69] where this risk was assessed as low. 
Table 5 Quality indicators assessed in study outcomes- included studies $(n=12)$

\begin{tabular}{|c|c|c|c|c|c|c|}
\hline \multirow[t]{2}{*}{ First author (year) } & \multicolumn{6}{|c|}{ Quality indicators assessed in study outcomes } \\
\hline & Effectiveness & Efficiency & Timeliness & Safety \& risk & Equity & Person \& family centred care \\
\hline \multicolumn{7}{|c|}{ - Discharge protocol \& advanced practice nurse } \\
\hline Naylor (1990) [72] & $\checkmark$ & $\checkmark$ & & $\checkmark$ & & \\
\hline Naylor (1994) [68] & $\checkmark$ & $\checkmark$ & & $\checkmark$ & & \\
\hline Naylor (1999) [77] & $\checkmark$ & $\checkmark$ & & $\checkmark$ & & $\checkmark$ \\
\hline Naylor (2004) [75] & $\checkmark$ & $\checkmark$ & & $\checkmark$ & & $\checkmark$ \\
\hline Enguidanos (2012) [74] & $\checkmark$ & $\checkmark$ & & $\checkmark$ & & $\checkmark$ \\
\hline \multicolumn{7}{|c|}{ - General practitioner and primary care nurse models } \\
\hline Weinberger (1996) [67] & $\checkmark$ & $\checkmark$ & & $\checkmark$ & & $\checkmark$ \\
\hline Mclnnes (1999) [73] & $\checkmark$ & $\checkmark$ & & $\checkmark$ & $\checkmark$ & $\checkmark$ \\
\hline Preen (2005) [66] & $\checkmark$ & $\checkmark$ & $\checkmark$ & $\checkmark$ & $\checkmark$ & $\checkmark$ \\
\hline \multicolumn{7}{|c|}{ - Self-management and transitional coaching } \\
\hline Coleman (2006) [69] & $\checkmark$ & $\checkmark$ & & $\checkmark$ & & \\
\hline \multicolumn{7}{|c|}{ - Discharge case management } \\
\hline $\operatorname{Lim}(2003)[76]$ & $\checkmark$ & $\checkmark$ & & $\checkmark$ & & \\
\hline \multicolumn{7}{|c|}{ - Inpatient geriatric evaluation, co-management and transitional care } \\
\hline Hansen (1995) [70] & $\checkmark$ & $\checkmark$ & & $\checkmark$ & $\checkmark$ & \\
\hline Legrain (2011) [71] & $\checkmark$ & $\checkmark$ & & $\checkmark$ & & \\
\hline
\end{tabular}

There was a low risk of reporting bias, bias associated with the selection of particular outcomes for reporting [38], across 11 of the 12 studies with one study providing insufficient information to make an assessment [68]. The potential for other sources of bias was assessed as low risk in three studies $[69,74,75]$ and unclear across the remaining nine studies. Findings from the bias assessment of the 12 studies are presented in Table 6 .

\section{Discussion}

This review synthesised evidence about the quality of transitional care for older people transitioning from hospital to home in order to produce recommendations for research and practice.

Transitional care interventions examined in the 12 studies were conducted by a range of health and social care professionals, and by older people including advanced practice nurses [68,72,74,75,77], general practitioners and practice nurses $[66,67,73]$, the older person and their carer with support from a transition coach [69], case managers [76] and geriatricians [70,71]. This indicates that transitional care can be undertaken by a range of health professonal disciplines and importantly, by older people and carers themselves with appropriate support.

Numerous outcomes were assessed [66-77] with mixed findings. Results from the included studies indicate that, except for general practitioner and practice nurse interventions, transitional care delayed and prevented early re-hospitalization. Outcome data in relation to length of stay, costs and quality of life were inconclusive. Notably, a recent Cochrane systematic review by Shepperd et al. [12] found transitional care resulted in cost shifting from the acute to community sector rather than a reduction in costs for the health system as a whole.

Findings indicate that general practitioner and practice nurse interventions were not effective in reducing rehospitalization rates $[67,73]$ or length of stay $[66,73]$. The study by Weinberger et al. [67] found higher rates of re-hospitalization following their intervention and if re-admitted, the veterans in their study had longer stays in hospital. The veterans who participated in this study also reported low quality of life and may have been in particularly poor health at discharge. It is possible that the transitional care intervention resulted in earlier identification of ill health among these participants with subsequent re-hospitalization [67]. No other included studies targeted veterans and findings from this study may be limited in generalizability to older US veterans. Findings of McInnes et al. [73] and Preen et al. [66] were difficult to interpret because in both studies the intervention was not fully implemented. Only $42 \%$ of general practitioners contributed to the discharge plan in the study by Preen et al. [66] and only 52\% of patients had general practitioner input into their discharge plan in the study by McInnes et al. [73]. The low rates of participation by general practitioners in both studies highlights the challenges associated with additional work responsibilities in transitional care for primary care providers and 
Table 6 Bias assessment - included studies $(n=12)$

\begin{tabular}{|c|c|c|c|c|c|c|c|}
\hline \multirow[b]{3}{*}{ First author (year) } & \multicolumn{7}{|l|}{ Risk of bias } \\
\hline & \multicolumn{2}{|l|}{ Selection bias $^{1}$} & \multirow{2}{*}{$\begin{array}{l}\text { Performance bias } \\
\text { Blinding of participants } \\
\text { and personnel }\end{array}$} & \multirow{2}{*}{$\begin{array}{l}\text { Detection bias }{ }^{3} \\
\text { Blinding of outcome assessment }\end{array}$} & \multirow{2}{*}{$\begin{array}{l}\text { Attrition bias }{ }^{4} \\
\text { Incomplete outcome } \\
\text { data }\end{array}$} & \multirow{2}{*}{$\begin{array}{l}\text { Reporting bias }{ }^{5} \\
\text { Selective reporting }\end{array}$} & \multirow{2}{*}{$\begin{array}{l}\text { Other bias } \\
\text { Other sources } \\
\text { of bias }\end{array}$} \\
\hline & $\begin{array}{l}\text { Random sequence } \\
\text { generation }\end{array}$ & $\begin{array}{l}\text { Allocation } \\
\text { concealment }\end{array}$ & & & & & \\
\hline Coleman (2006) [69] & Low risk & Unclear risk & Unclear risk & Low risk & Low risk & Low risk & Low risk \\
\hline Enguidanos (2012) [74] & Low risk & Low risk & Unclear risk & Low risk & $\begin{array}{l}\text { High risk (self-efficacy, service } \\
\text { satisfaction) Low risk (service } \\
\text { utilisation) }\end{array}$ & Low risk & Low risk \\
\hline Hansen (1995) [70] & Low risk & Unclear risk & $\begin{array}{l}\text { High risk (personnel) } \\
\text { Unclear risk (participants) }\end{array}$ & Unclear risk & Unclear risk & Low risk & Unclear risk \\
\hline Legrain (2011) [71] & Low risk & Low risk & $\begin{array}{l}\text { High risk (personnel) } \\
\text { Unclear risk (participants) }\end{array}$ & Unclear risk & Unclear risk & Low risk & Unclear risk \\
\hline $\operatorname{Lim}(2003)$ [76] & Low risk & Low risk & Unclear risk & Low risk & Unclear risk & Low risk & Unclear risk \\
\hline Mclnnes (1999) [73] & Low risk & Low risk & Unclear risk & $\begin{array}{l}\text { Low risk (service utilisation data) } \\
\text { Unclear risk (questionnaire data) }\end{array}$ & Unclear risk & Low risk & Unclear risk \\
\hline Naylor (1990) [72] & Low risk & Low risk & $\begin{array}{l}\text { High risk (personnel) } \\
\text { Unclear risk (participants) }\end{array}$ & Unclear risk & Unclear risk & Low risk & Unclear risk \\
\hline Naylor (1994) [68] & Unclear risk & Unclear risk & Unclear risk & Unclear risk & Unclear risk & Unclear risk & Unclear risk \\
\hline Naylor (1999) [77] & Low risk & Low risk & $\begin{array}{l}\text { High risk (personnel) } \\
\text { Unclear risk (participants) }\end{array}$ & Low risk & Unclear risk & Low risk & Unclear risk \\
\hline Naylor (2004) [75] & Low risk & Low risk & $\begin{array}{l}\text { High risk (personnel) } \\
\text { Unclear risk (participants) }\end{array}$ & Low risk & Unclear risk & Low risk & Low risk \\
\hline Preen (2005) [66] & Unclear risk & Unclear risk & Unclear risk & Unclear risk & Unclear risk & Low risk & Unclear risk \\
\hline Weinberger (1996) [67] & Unclear risk & Low risk & Unclear risk & Low risk & Unclear risk & Low risk & Unclear risk \\
\hline
\end{tabular}

${ }^{1}$ Selection bias refers to the adequacy of randomisation processes (random sequence generation) and the adequacy of the concealment of allocation to intervention group (allocation concealment) [38] ${ }^{2}$ Performance bias is the knowledge of research participants and research staff of the interventions that participants were allocated to [38].

${ }^{3}$ Detection bias is outcome assessors' knowledge of the interventions that participants were allocated to [38].

${ }^{4}$ Attrition bias was the potential for biased conclusions resulting from incomplete outcome data [38].

${ }^{5}$ Reporting bias referred to the selection of particular outcomes for reporting [38].

${ }^{6}$ The potential for other sources of bias (other bias) was also appraised [38] 
suggest that in these two studies the intervention was not feasible for general practitioners. Additionally, the sample size was insufficient to detect an intervention effect in the study by Preen et al. [66].

Effectiveness in terms of symptom management was not specifically studied as an outcome/s in the included studies. This is of concern given findings from two Australian descriptive studies [78,79] where people reported symptom exacerbation at discharge and an absence of assistance with symptom management and functioning in relation to pain, fatigue, loss of mobility, and grief during care transitions from hospital to home.

Results [66-77] also highlighted the potential for transitional care to result in improved satisfaction for older people however caregiver satisfaction has not been measured. There is also limited understanding of the burden to caregivers.

Timeliness, equity and access are described as part of the intervention in each included study [66-77]. However, consistent with the findings from other research [10,27] outcomes assessing timeliness, equity and access have not been clearly reported in this research.

Research included in this systematic review [66-77] suggests that measures of re-hospitalization rates or length of stay have been consistently studied in the general transitional care experimental research since 1990, indicating an outcome focus on select quality elements related to effectiveness, safety and efficiency for inpatient services. Other indicators of quality in transitional care, as recommended by the Institute of Medicine [4], Department of Health [8], and Australian Commission on Quality and Safety in Healthcare [6], have not been a consistent outcome focus, suggesting gaps in understanding about timeliness, equity, family/carer centred care and symptom management for older people [10,27].

Person and family centred care is described as a part of interventions in the included studies [66-77]. Reporting of outcome measures of person centred care has been focussed on patient satisfaction. Six of the twelve identified studies assessed patient satisfaction following the transitional care intervention $[66,67,73-75,77]$. Only two studies included carer burden $[68,76]$. There was little evidence about the 'experience' of older people and their family/ carers although qualitative studies $[2,78,80,81]$ have described problems and unmet needs from older peoples' perspectives and experiences associated with ineffective transitional care. No studies specifically assessed emotional support for older people and their families/carers.

Although self-management and education were described as components of interventions in the included studies in particular in the intervention by Coleman and associates [64,69], outcome evidence about self-care and self-management related to older people and their carers' use of the health care system was limited.
These results are of particular interest because older people and their families/carers are increasingly expected to self-care at home following early discharge and they are expected to navigate complex health care systems $[3,13,23]$. Although Coleman and colleagues [64,69] conducted focus groups to ascertain what older people wanted in transitional care, no other study contained reports about the involvement of older people and their carers/family in the design of the transitional care intervention that they tested.

In other literature, Naylor [82] and Bauer et al. [10] found that many older people and their family/carers reported unmet discharge needs about information and access to services in the community, and they were not involved in discharge related decisions. Additionally, Bauer et al. [10] found that family carers reported frustration with discharge planning processes, lack of information and poor communication with health practitioners.

\section{Bias assessment}

Findings were mixed in relation to potential sources of bias across the 12 studies. Over $40 \%$ of articles did not provide adequate information to accurately assess the risk of bias, suggesting a need for improved reporting about how methods were implemented and about how attrition of participants was managed. Overall, there was low risk of selection bias, however some studies provided insufficient information to assess this risk in relation to the randomisation process [66-68], or how allocation concealment was undertaken [66,68-70]. No studies were identified to have low risk in regard to performance bias therefore there is potential risk of bias in this regard [38]. Notably, blinding of personnel to group allocation would not be possible for complex health and social care interventions such as transitional care as practitioners conducting the intervention would be aware that they were doing so. No studies reported on the blinding of participants, therefore the risk of performance bias in relation to participants is not known. Of the 12 studies, five provided insufficient information to assess the risk of detection bias [66,68,70-72]. The reporting of missing data and how these data were managed was mixed across the 12 studies, indicating unclear risk and potential for attrition bias. There was a low risk of reporting bias across the 12 studies with only one study providing insufficient information to make an assessment [68]. The potential for other sources of bias was assessed as low risk in three studies [69,74,75] and unclear across the remaining nine studies.

\section{Limitations of the current evidence base}

In all studies, the transitional care intervention was compared with standard hospital discharge. However, standard hospital discharge was not clearly described and it was therefore not known what the comparison 
control conditions entailed. All studies included description of interventions in terms of particular quality indicators such as person and family centred care and timeliness, however with exception to patient satisfaction surveys conducted in half of the studies, there was limited reporting of outcome assessment of these quality indicators.

\section{Limitations of the review}

Older people included in the review comprised those aged over 60 years. This potentially includes a wide range in age and a group of people with different health needs. The average ages of people in the studies were specified to provide more focused information. Additionally, the review did not capture grey literature, publically available literature not published in peer review journals; therefore all relevant research may not have been included. Only English language publications were included, therefore the review synthesises the best available evidence published in English only.

\section{Conclusion}

Despite these limitations, findings from this review suggest that there are gaps in the evidence base regarding the quality of transitional care interventions for older people and their families/carers where quality is assessed in terms of effective, efficient, safe and low risk, timely, equitable and person and family centred care. There is a need for improved understanding and evidence about the quality of transitional care for older people and their carers in particular domains of person and family centred care; the patient and carer experience, carer burden and support, and emotional support for older people and their carers during care transitions. This is of particular concern as older people and their families/carers are discharged early and expected to self-care and navigate complex and fragmented systems of care independently. There is a need for improved understanding about outcomes in relation to equity and timeliness in care transitions for older people and their carers. The results from this review highlight that self-management and health outcomes including those assessing symptom management require stronger focus in this literature.

In view of the changing health care context and dehospitalisation of health and aged care, and because care transitions are increasingly complex, the results also suggest there is a need for research that involves the person and their family/caregiver in the design of high quality transition care interventions in order to meet the needs of older people and their families/carers. The shift in responsibility for health and aged care from acute inpatient settings to the community sector and to family and carers means that older people and their families should be involved in planning and decisions about their care and identifying what would be of most assistance to them.

\section{Additional file}

Additional file 1: Data extraction tool

Competing interests

The authors declare that they have no competing interests.

\section{Authors' contributions}

JA conceptualised and designed the review, collected, screened and coded all studies (data), completed data analysis and interpretation, and drafted the manuscript. PML, AMH, and RB contributed to the conceptualisation and design of the review, participated in the screening process, assessed studies for risk of bias and critically reviewed and revised the manuscript for intellectual content. All authors read and approved the final manuscript.

\section{Author details}

'Deakin University, School of Nursing and Midwifery, 221 Burwood Hwy, Burwood 3125, Vic, Australia. ${ }^{2}$ Deakin University, School of Nursing and Midwifery; Centre for Nursing Research - Deakin University and Monash Health Partnership, Monash Health, 221 Burwood Hwy, Burwood 3125, Vic, Australia. ${ }^{3}$ Deakin University, Faculty of Health \& School of Nursing and Midwifery, 221 Burwood Hwy, Burwood 3125, Vic, Australia.

Received: 18 March 2014 Accepted: 1 August 2014 Published: 15 August 2014

\section{References}

1. Anderson G: Chronic Care: Making the case for ongoing care. NJ USA; 2010 (http://www.rwjf.org/content/dam/farm/reports/reports/2010/ rwjf54583).

2. Naylor M, Hirschman K, O'Connor M, Barg R, Pauly M: Engaging older adults in their transitional care: what more needs to be done? J Comp Eff Res 2013, 2(5):457-468

3. Ham C, Imison C, Goodwin N, Dixon A, South P: Where next for the NHS reforms? The case for integrated care. London: The King's Fund; 2011.

4. Institute of Medicine: Crossing the quality quasm: A new health system for the 21st century. Washington: Institute of Medicine; 2001.

5. National Health and Hospitals Reform Commission: A Healthier Future for all Australians - Final Report of the National Health and Hospitals Reform Commission. Canberra, ACT: Commonwealth of Australia; 2009.

6. Australian Commission on Quality and Safety in Healthcare: National safety and quality health service standards. Sydney: ACQSH; 2011.

7. Beattie M, Shepherd A, Howieson B: Do the Institute of Medicine's (IOM's) dimensions of quality capture the current meaning of quality in health care? - An integrative review. J Res Nurs 2013, 18(4):288-304.

8. Department of Health: Quality governence in the NHS-A guide for provider boards. London: Department of Health; 2011.

9. Allen J, Ottmann G, Roberts G: Multi-professional communication for older people in transitional care: a review of the literature. Int J Older People Nurs 2013, 8(4):253-269.

10. Bauer M, Fitzgerald L, Haesler E, Manfrin M: Hospital discharge planning for frail older people and their family. Are we delivering best practice? A review of the evidence. J Clin Nurs 2009, 18(18):2539-2546.

11. Laugaland $K$, Aase $K$, Barach P: Interventions to improve patient safety in transitional care - a review of the evidence. Work 2012, 41(S1):2915-2924

12. Shepperd S, Lannin NA, Clemson LM, McCluskey A, Cameron ID, Barras SL: Discharge planning from hospital to home. In The Cochrane Database of Systematic Reviews. London: 2013. http://summaries.cochrane.org/ CD000313/discharge-planning-from-hospital-to-home.

13. Productivity Commission: Caring for Older Australians (Vol. 1). Canberra, Australia: Productivity Commission: 201.

14. Australian Institute of Health and Welfare: Older Australia at a glance: 4th edition. Canberra, Australia: Australian Institute of Health and Welfare; 2007.

15. Australian Institute of Health and Welfare: Australia's Health 2012. Canberra, Australia: Australian Insitute of Health and Welfare; 2012.

16. Uhlenberg P: Demography is not destiny: The challenges and opportunities of global population aging. Generations 2013, 37(1):12-18.

17. National Centre for Social and Economic Modelling: Who's Going to Care? Informal Care and an Ageing Population. Canberra, Australia: National Centre for Social and Economic Modelling; 2004. 
18. Runge C, Gilham J, Peut A: Transitions in Care of People with Dementia: A Systematic Review of the Literature. Sydney, Australia: University of New South Wales; 2009

19. Johnstone $\mathrm{M}$, Kanitsaki $\mathrm{O}$ : Ethnic aged discrimination and disparities in health and social care: a question of social justice. Australas J Ageing 2008, 27(3):110-115.

20. Coleman EA, Boult C: Improving the quality of transitional care for persons with complex care needs. J Am Geriatr Soc 2003, 51(4):556-557.

21. Holland DE, Harris MR: Discharge planning, transitional care, coordination of care, and continuity of care: clarifying concepts and terms from the hospital perspective. Home Health Care Serv Q 2007, 26(4):3-19.

22. Naylor MD: Transitional care: a critical dimension of the home healthcare quality agenda. J Healthc Qual 2006, 28(1):48-54.

23. Naylor M, Keating SA: Transitional care. J Soc Work Educ 2008, 44(Supplement):65-73.

24. Naylor MD, Aiken LH, Kurtzman ET, Olds DM, Hirschman KB: The Importance Of Transitional Care In Achieving Health Reform. Health Aff 2011, 30(4):746-754.

25. Reed J, Cook G, Childs S, McCormack B: A literature review to explore integrated care for older people. Int J Integrated Care 2005, 5(January):1-10.

26. Harrison PL, Hara PA, Pope JE, Young MC, Rula EY: The impact of postdischarge telephonic follow-up on hospital readmissions. Popul Health Manag 2011, 14(1):27-32.

27. Mansah M, Griffith R, Fernandez R, Chang E: Effectiveness of strategies to promote safe transition of elderly people across care settings (systematic review). In The Joanna Briggs Institute Systematic Reviews. Adelaide, Australia: 2000. http://www.joannabriggs.edu.au/pubs/ systematic_reviews.php.

28. Coleman EA: Falling through the cracks: challenges and opportunities for improving transitional care for persons with continuous complex care needs. J Am Geriatr Soc 2003, 51(4):549-555.

29. Naylor MD, Kurtzman ET, Grabowski DC, Harrington C, McClellan M, Reinhard SC: Unintended consequences of steps to cut readmissions and reform payment may threaten care of vulnerable older adults. Health Aff 2012, 31(7):1623-1632.

30. Parker S, Lee S, Fadayevatan R: Co-ordinating discharge of elderly people from hospital to the community. Evid base Healthc Publ Health 2004, 8(6):332-334.

31. Hyde C, Robert I, Sinclair A: The effects of supporting discharge from hospital to home in older people. Age Ageing 2000, 29(3):271-279.

32. Jackson M: Discharge planning: issues and challenges for gerontological nursing. A critique of the literature. J Adv Nurs 1994, 19(3):492-502.

33. Mistaien P, Francke A, Poot E: Interventions aimed at reducing problems in adult patients discharged from hospital to home: systematic meta-review. BMC Health Serv Res 2007, 7:47.

34. Sunil K, Frank LF, Christopher P, Mark W, Preetha B, David B: Deficits in communication and information transfer between hospital-based and primary care physicians: implications for patient safety and continuity of care. JAMA 2007, 297(8):831-841.

35. Coffey A: Discharging older people from hospital to home: what do we know? Int J Older People Nurs 2006, 1(3):141-150.

36. Closs S: Discharge communications between hospital and community health care staff: a selective review. Health Soc Care Community 1997, 5(3):181-197.

37. Payne S, Kerr C, Hawker S, Hardey M, Powell J: The communication of information about older people between health and social care practitioners. Age Ageing 2002, 31(2):107-117.

38. Higgins J, Green S: Cochrane handbook for systematic reviews of interventions. In The Cochrane Collaboration. London: 2011. http://handbook.cochrane.org.

39. Cioffi J, Wilkes L, Warne B, Harrison K, Vonu-Boriceanu O: Community nursing care for clients with chronic and complex conditions. Collegian 2007, 14(4):21-25.

40. Kemp L, Harris $\mathrm{E}$, Comino E: Changes in community nursing in Australia: 1995-2000. J Adv Nurs 2005, 49(3):307-314

41. World Health Organisation: Definition of an older or elderly person. Geneva: World Health Organisation; 2013. http://www.who.int/healthinfo/survey/ ageingdefnolder/en/index.html.

42. Thomson Reuters: Endnote version 16. USA: 2013. http://endnote.com.

43. Arbaje Al, Maron DD, Yu Q, Wendel VI, Tanner E, Boult C, Eubank KJ, Durso SC: The Geriatric Floating Interdisciplinary Transition Team. J Am Geriatr Soc 2010, 58(2):364-370.
44. Bull MJ, Hansen HE, Gross CR: A professional-patient partnership model of discharge planning with elders hospitalized with heart failure. Appl Nurs Res 2000, 13(1):19-28.

45. Balaban R, Weissman J, Samuel P, Woolhandler S: Redefining and redesigning hospital discharge to enhance patient care: a randomized controlled study. J Gen Intern Med 2008, 23(8):1228-1233.

46. Bonnet-Zamponi D, D'Arailh L, Konrat C, Delpierre C, Lieberherr D, Lemaire A, Tubach F, Lacaille S, Legrain S: Drug-related readmissions to medical units of older adults discharged from acute geriatric units: Results of the Optimization of Medication in AGEd Multicenter randomized controlled trial. J Am Geriatr Soc 2013, 61(1):113-121.

47. Brand CA, Jones CT, Lowe AJ, Nielsen DA, Roberts CA, King BL, Campbell DA: A transitional care service for elderly chronic disease patients at risk of readmission. Aust Health Rev 2004, 28(3):275-284.

48. Coleman EA, Smith JD, Frank JC, Min S, Parry C, Kramer AM: Preparing patients and caregivers to participate in care delivered across settings: the care transitions intervention. J Am Geriatr Soc 2004, 52(11):1817-1825.

49. Dedhia P, Kravet S, Bulger J, Hinson T, Sridharan A, Kolodner K, Wright S, Howell E: A quality improvement intervention to facilitate the transition of older adults from three hospitals back to their homes. J Am Geriatr Soc 2009, 57(9):1540-1546.

50. Einstadter D, Cebul R, Franta P: Effect of a nurse case manager on post discharge follow-up. J Gen Intern Med 1996, 11(11):684-688.

51. Golden AG, Tewary S, Dang S, Roos BA: Care management's challenges and opportunities to reduce the rapid rehospitalization of frail community-dwelling older adults. Gerontologist 2010, 50(4):451-458.

52. Haggmark C, Nilsson B: Effects of an intervention programme for improved discharge-planning. Nordic Journal of Nursing Research \& Clinical Studies / Vård i Norden 1997, 17(2):4-8.

53. Hansen FR, Spedtsberg K, Schroll M: Geriatric follow-up by home visits after discharge from hospital: a randomized controlled trial. Age Ageing 1992, 21(6):445-450.

54. Hébert R, Dubois M, Raiche M, Dubuc N: The effectiveness of the PRISMA integrated service delivery network: preliminary report on methods and baseline data. Int J Integr Care 2008, 8(Jan-Mar):1-16.

55. Hébert R, Veil A, Raiche M, Dubois M, Dubuc N, Tousignant M: Evaluation of the implementation of PRISMA, a coordination-type integrated service delivery system for frail older people in Québec. Journal of Integrated Care 2008, 16(6):4-14

56. Hegney D, McCarthy A, de la Rue MB, Fahey P, Gorman D, Martin-McDonald $K$, Pretty G, Sundin-Huard D: Discharge planning from the acute sector for people over the age of 65. Collegian 2002, 9(3):15-21.

57. Jack BW, Chetty VK, Anthony D, Greenwald JL, Sanchez GM, Johnson AE, Forsythe SR, O'Donnell AK, Paasche-Orlow MK, Manasseh C, Martin S, Culpepper $\mathrm{L}$ : A reengineered hospital discharge program to decrease rehospitalization: a randomized trial. Ann Intern Med 2009, 150(3):178-187.

58. Jeangsawang N, Malathum P, Panpakdee O, Brooten D, Nityasuddhi D: Comparison of outcomes of discharge planning and post-discharge follow-up care, provided by advanced practice, expert-by-experience, and novice nurses, to hospitalized elders with chronic healthcare conditions. Int J Res Nurs 2012, 16(4):343-360.

59. Lattimer C: Practices to improve transitions of care: a national perspective. N C Med J 2012, 73(1):45-47.

60. Melton LD, Foreman C, Scott E, McGinnis M, Cousins M: Prioritized post-discharge telephonic outreach reduces hospital readmissions for select high-risk patients. Am J Manag Care 2012, 18(12):838-846.

61. O'Reilly J, Lowson K, Green J, Young JB, Forster A: Post-acute care for older people in community hospitals-a cost-effectiveness analysis within a multi-centre randomised controlled trial. Age Ageing 2008, 37(5):513-520.

62. Ornstein K, Smith KL, Foer DH, Lopez-Cantor MT, Soriano T: To the hospital and back home again: A nurse practitioner-based transitional care program for hospitalized homebound people. J Am Geriatr Soc 2011 59(3):544-551

63. Parker $\mathrm{S}$, Oliver $\mathrm{P}$, Pennington $\mathrm{M}$, Bond J, Jagger $\mathrm{C}$, Enderby $\mathrm{P}$, Curless $\mathrm{R}$, Chater T, Vanoli A, Fryer K, Cooper C, Julious S, Donaldson C, Dyer C, Wynn T, John A, Ross D: Rehabilitation of older patients: day hospital compared with rehabilitation at home. A randomised controlled trial. Health Tech Assess 2009, 13(39):1-168.

64. Parry C, Mahoney E, Chalmers SA, Coleman EA: Assessing the quality of transitional care: further applications of the care transitions measure. Med Care 2008, 46(3):317-322. 
65. Steeman E, Moons P, Milisen K, De Bal N, De Geest S, De Froidmont C, Tellier V, Gosset C, Abraham I: Implementation of discharge management for geriatric patients at risk of readmission or institutionalization. Int $J$ Qual Health Care 2006, 18(5):352-358.

66. Preen DB, Bailey BES, Wright A, Kendall P, Phillips M, Hung J, Hendriks R, Mather A, Williams E: Effects of a multidisciplinary, post-discharge continuance of care intervention on quality of life, discharge satisfaction, and hospital length of stay: a randomized controlled trial. Int I Qual Health Care 2005, 17(1):43-51.

67. Weinberger M, Oddone EZ, Henderson WG: Does increased access to primary care reduce hospital readmissions? New Engl J Med 1996, 334(22):1441-1447.

68. Naylor M, Brooten D, Jones R, Lavizzo-Mourey R, Mezey M, Pauly M: Comprehensive discharge planning for the hospitalized elderly. A randomized clinical trial. Ann Intern Med 1994, 120(12):999-1006.

69. Coleman EA, Parry C, Chalmers S, Min SJ: The care transitions intervention: results of a randomized controlled trial. Arch Intern Med 2006, 166(17):1822-1828.

70. Hansen FR, Poulsen $\mathrm{H}$, Sørensen KH: A model of regular geriatric follow-up by home visits to selected patients discharged from a geriatric ward: a randomized controlled trial. Aging 1995, 7(3):202-206.

71. Legrain S, Tubach F, Bonnet-Zamponi D, Lemaire A, Aquino J-P, Paillaud E, Taillandier-Heriche E, Thomas C, Verny M, Pasquet B, Moutet Aline L, Lieberherr $D$, Lacaille S: A new multimodal geriatric discharge-planning intervention to prevent emergency visits and rehospitalizations of older adults: The Optimization of Medication in AGEd multicenter randomized controlled trial. J Am Geriatr Soc 2011, 59(11):2017-2028.

72. Naylor MD: Comprehensive discharge planning for hospitalized elderly: a pilot study. Nurs Res 1990, 39(3):156-161.

73. Mclnnes E, Mira M, Atkin N, Kennedy P, Cullen J: Can GP input into discharge planning result in better outcomes for the frail aged: results from a randomized controlled trial. Fam Pract 1999, 16(3):289-293.

74. Enguidanos S, Gibbs N, Jamison P: From hospital to home: a brief nurse practitioner intervention for vulnerable older adults. Journal of Gerontological Nursing 2012, 38(3):40-50.

75. Naylor MD, Brooten DA, Campbell RL, Maislin G, McCauley KM, Schwartz JS: Transitional care of older adults hospitalized with heart failure: a randomized, controlled trial. J Am Geriatr Soc 2004, 52(5):675-684.

76. Lim W, Lambert S, Gray L: Effectiveness of case management and post-acute services in older people after discharge. Med J Aust 2003, 178(6):262-266.

77. Naylor MD, Brooten D, Campbell R, Jacobsen BS, Mezey MD, Pauly MV, Schwartz JS: Comprehensive discharge planning and home follow-up of hospitalized elders. JAMA 1999, 281(7):613-620.

78. Grimmer K, Moss J, Falco J: Experiences of elderly patients regarding independent community living after discharge from hospital: a longitudinal study. International J Qual Health Care 2004, 16(6):465-472.

79. Pearson P, Procter S, Wilcockson J, Allgar V: The process of hospital discharge for medical patients: a model. J Adv Nurs 2004, 46(5):496-505.

80. Graham CL, Ivey SL, Neuhauser L: From hospital to home: assessing the transitional care needs of vulnerable seniors. Gerontologist 2009, 49(1):23-33.

81. Rydeman I, Törnkvist $L$ : Getting prepared for life at home in the discharge process-From the perspective of the older persons and their relatives. Int J Older People Nurs 2010, 5(4):254-264.

82. Naylor MD: Transitional care of older adults. Annu Rev Nurs Res 2002, 20(1):127-147.

\section{Submit your next manuscript to BioMed Central and take full advantage of:}

- Convenient online submission

- Thorough peer review

- No space constraints or color figure charges

- Immediate publication on acceptance

- Inclusion in PubMed, CAS, Scopus and Google Scholar

- Research which is freely available for redistribution

Submit your manuscript at www.biomedcentral.com/submit 